Annuaire suisse de politique de développement

16 | 1997

Environnement et développement, 5 ans après Rio

\title{
3. Politique intérieure et extérieure
}

\section{(2) OpenEdition}

1 Journals

Édition électronique

URL : http://journals.openedition.org/aspd/789

DOI : 10.4000/aspd.789

ISSN : 1663-9669

Éditeur

Institut de hautes études internationales et du développement

\section{Édition imprimée}

Date de publication : 1 mars 1997

Pagination : 93-111

ISSN : 1660-5934

\section{Référence électronique}

«3. Politique intérieure et extérieure », Annuaire suisse de politique de développement [En ligne], 16 |

1997, mis en ligne le 07 août 2012, consulté le 08 septembre 2020. URL : http://

journals.openedition.org/aspd/789; DOI : https://doi.org/10.4000/aspd.789 


\section{POLITIOUE INTÉRIEURE ET EXTÉRIEURE}

\section{POLITIQUE D'ASILE ET QUESTIONS MIGRATOIRES}

La politique d'asile suisse se fonde sur la loi sur l'asile de 1979. Depuis son entrée en vigueur, celle-ci a déjà fait l'objet de quatre révisions partielles destinées à prendre en compte les changements intervenus dans le domaine de l'asile. Une révision totale de la loi est actuellement en préparation. Elle est avant tout motivée par la volonté de rendre la pratique de l'asile plus restrictive, mais sa principale innovation réside dans la création d' une base légale permettant d'accorder une protection provisoire à des réfugiés dits de la violence.

Ces dix dernières années, le nombre des demandes d'asile déposées en Suisse a fluctué entre 8000 et 42'000 par an. Leur nombre a atteint 17'021 en 1995 et 11 '069 de janvier à la fin août 1996.

Lors de la votation du 1er décembre 1996, le peuple a rejeté par 53,6\% l' initiative « contre l'immigration clandestine». La Confédération s'efforce actuellement de formuler une politique migratoire dans le but de rédiger une loi sur les migrations, qui prendrait en considération non seulement les causes et les conditions de la fuite, mais aussi la conjoncture économique, la situation sur le marché suisse du travail et l'état des finances fédérales. On souhaite en fait élaborer une politique permettant de freiner les migrations et améliorer la coordination entre les services politiques concernés par l'immigration.

La guerre, les conflits armés et la misère économique sont les principaux motifs de fuite pour la plupart des quelques 50 millions de réfugiés de par le monde. Les grands flux migratoires touchent les pays en développement et seule une part infime de cette migration parvient jusque dans les pays industrialisés. L'immense majorité des réfugiés restent au Sud, aggravant les problèmes de développement de la région où ils sont stationnés, comme le souligne à plusieurs reprises le Conseil fédéral dans le cadre de la coopération au développement et de l'aide humanitaire. 'Pour ce qui est de la Suisse, la majeure partie des requérants d'asile proviennent des zones en guerre de l'ex-Yougoslavie, du Sri Lanka et de la Turquie.

\section{$\square$ Demandes d'asile}

Le tableau 15 indique qu'avec ses 17'021 demandes d'asile l'année 1995 compte parmi celles qui ont connu un afflux de réfugiés relativement faible. Les années 1990 et 1991 font exception, car les requêtes émises par des personnes en provenance des zones en guerre de l'ex-Yougoslavie sont venues grossir le nombre des demandes.

\footnotetext{
Voir p. ex. le chapitre Les mouvements migratoires dans le Message concernant la continuation de la coopération technique et de l'aide financière en faveur des pays en développement du 20.4.1994. La politique migratoire, la coopération au développement, ainsi que le rapport de Peter Arbenz ont fait l'objet de commentaires détaillés dans l'Annuaire 1996 (p. 95 et suiv.).
} 


\section{Tableau n⿳0 15}

\begin{tabular}{|c|c|c|c|c|c|c|c|c|c|c|}
\hline \multicolumn{11}{|c|}{ Demandes d'asile déposées en Suisse, 1985 à 1995} \\
\hline \multicolumn{11}{|c|}{ (nombre de requérants) } \\
\hline 1985 & 1986 & 1987 & 1988 & 1989 & 1990 & 1991 & 1992 & 1993 & 1994 & 1995 \\
\hline 9703 & 8546 & $10 ’ 913$ & $16^{\prime} 726$ & $24 ’ 425$ & $35^{\prime} 836$ & $41^{\prime} 692$ & 17960 & $24 ’ 739$ & $16^{\prime} 134$ & $17^{\prime} 021$ \\
\hline
\end{tabular}

\begin{tabular}{lllr}
\hline Pays de provenance des requérants en $\mathbf{1 9 9 5}$ & & (nombre de requérants) \\
\hline Rép. féd. de Yougoslavie & 5491 & Sri Lanka & 1024 \\
\hline Bosnie-Herzégovine & 3534 & Angola & 493 \\
\hline Turquie & 1293 & Somalie & 478 \\
\hline
\end{tabular}

Source : DFJP/Office fédéral des réfugiés.

La plupart des demandes émanent de personnes qui fuient des régions en guerre : ex-Yougoslavie, Turquie (conflit entre l'Etat et les Kurdes), Sri Lanka ; ainsi que des pays africains secoués par des troubles politiques (Angola, Somalie, Zaïre, Algérie).

Le taux d'acceptation moyen a atteint $14,9 \%$ en 1995 et $12,5 \%$ en 1994 .

A la fin de l'année 1995, 125448 personnes séjournaient en Suisse au titre de la procédure d'asile, dont 24581 étaient des réfugiés reconnus. Parmi tous les requérants et toutes les personnes provisoirement admises, seuls $35 \%$ ont un emploi. En 1995, les prestations d'assistance versées se sont montées à 812 millions de francs au total. Ce montant comprend l'argent versé aux requérants et aux réfugiés, les frais administratifs des cantons et diverses dépenses de l'Office fédéral des réfugiés.

\section{$\square$ Renvoi / Aide au retour}

Depuis 1984, environ 30000 Sri Lankais sont venus chercher refuge en Suisse. La plus grosse vague d'arrivants remonte aux années 1989 à 1991. En raison de la guerre civile au nord et à l'est de l'île, les requérants déboutés n'ont été rapatriés jusqu'au début de 1994 que s'ils avaient commis en Suisse des « délits ou abus de droit ou fait preuve d'un comportement asocial $\gg .^{2}$ En janvier 1994, la Suisse a conclu avec le Sri Lanka une convention de rapatriement limitée à deux ans, qui devait garantir aux requérants déboutés de pouvoir rentrer au pays « dans la sécurité et la dignité ».

Cette convention a été prolongée pour deux nouvelles années. Le nombre des Tamouls qui ont demandé à bénéficier de l'aide au rapatriement est resté inférieur aux prévisions de l'Office fédéral des réfugiés : en 1994 et 1995, 385 personnes au total sont retournées au Sri Lanka en vertu de cette convention et environ un tiers d'entre elles l'on fait à titre volontaire. ${ }^{3}$ Les rapatriements ont toutefois dus être provisoirement interrompus car la situation au Sri Lanka ne permettait plus d'assurer un retour conforme aux principes de la convention.

${ }^{2}$ Communiqué de presse de l'Office fédéral des réfugiés du 18.12.1995, La convention conclue avec le Sri Lanka au sujet du rapatriement des requérants d'asile déboutés est prolongée de deux ans.

3 L'Office fédéral des réfugiés a publié ces chiffres dans son communiqué de presse du 18.12.1995. 
Les autorités ont aussi mis à l'essai une forme d'aide au retour avec la Turquie : 100 personnes se voient rembourser leurs frais de voyage, ainsi qu'une contribution à la réintégration de 455 francs par mois pendant six mois ; elles reçoivent par ailleurs deux visites d'un représentant de l'Organisation internationale des migrations. De plus, un centre de conseils au rapatriement ayant fait ses preuves à Genève, on envisage d'ouvrir d'autres centres de ce type dans les cantons.

Le Haut commissariat des Nations Unies pour les réfugiés (HCR) s'est élevé contre la décision suisse de renvoyer chez eux des réfugiés angolais et bosniaques. La directrice du HCR a estimé d'une part que la Suisse a lancé beaucoup trop tôt ses opérations de rapatriement (assorties d'une aide au retour) vers la Bosnie. En effet, le Conseil fédéral a décidé en avril 1996 que 21'000 réfugiés bosniaques seraient contraints de retourner dans leur pays jusqu'en avril ou jusqu'en août 1997, tout en soulignant que la situation ferait l'objet d'un examen sur place en mars 1997 et que l'on déciderait alors du maintien des échéances prévues. Depuis la mi-août 1996, une aide opérationnelle au rapatriement vers la Bosnie est en place. Jusqu'à la fin de 1996, cette structure a incité plus de 2000 Bosniaques à retourner chez eux.

Le HCR jugeait d'autre part que la situation politique et les conditions de sécurité en Angola n'offraient pas de garanties suffisantes pour que les réfugiés puissent retourner dans le pays et a donc recommandé à la Suisse de remettre ses renvois à plus tard. Dans les deux cas, le Conseil fédéral s'en est tenu à sa politique du renvoi.

Dans le cas des requérants albanais du Kosovo déboutés, le Conseil fédéral a prolongé le délai de renvoi jusqu'à la fin mars 1997, car le gouvernement de Belgrade refuse d'accueillir ces réfugiés. En collaboration avec d'autres pays européens, la Suisse fait pression sur les Etats de l'ex-Yougoslavie pour qu'ils concluent des convention sur le rapatriement.

\section{$\square$ Initiative contre l' immigration clandestine}

Cette initiative de l'Union démocratique du centre (UDC) vise à réduire massivement le nombre des demandes d'asile en refusant d'entrer en matière sur les requêtes déposées par des personnes entrées clandestinement en Suisse. Cette mesure, toucherait $85 \%$ de tous les requérants d'asile. Notons que l'initiative a été lancée au début des années nonante, période à laquelle le nombre des demandes avait littéralement explosé. Depuis lors, elle a toutefois été dépassée par l'évolution de la situation, c'est pourquoi le Conseil fédéral s'est contenté de recommander son rejet.

Il préconise en effet une harmonisation de la politique d'asile au niveau international, notamment avec les pays de l'Union européenne. Il a déclaré que l'initiative risquait de museler beaucoup trop la politique d'asile suisse en ne laissant guère de place à une pratique de l'asile orientée sur l'avenir et coordonnée au niveau international, dans le cadre d'une politique migratoire globale. Le Conseil national a repoussé l'initiative par 139. voix contre 36 et le Conseil des Etats par 35 voix contre 3 . En votation populaire, l'initiative a été refusée par $53,9 \%$ des votants. Au niveau des cantons, le résultat fut encore plus serré : 11 cantons ont accepté l'initiative, contre 12 qui l'ont rejetée. 


\section{$\square$ Révision de la loi sur l'asile}

Voici les grandes lignes du projet de révision totale de la loi sur l'asile : nouvelles dispositions prévoyant l'accueil provisoire de personnes fuyant des zones en guerre (réfugiés de la violence) ; simplification des structures d'assistance et abaissement des frais d'assistance ; dispositions relatives à la protection des données.

Réfugiés de la violence : Le projet de révision de la loi sur l'asile du Conseil fédéral prévoit que le gouvernement pourra décider collectivement combien de personnes fuyant les affres de la guerre pourront venir se réfugier en Suisse et y obtenir une protection provisoire, sans devoir passer par la procédure individuelle de demande d'asile (chapitre 4 du projet de loi). Dans son message sur la révision totale de la loi sur l'asile, le Conseil fédéral déclare que le chapitre 4 constitue la clé de voûte du projet. Selon le gouvernement, la notion de réfugiés de la violence se fonde sur les éléments suivants :

1. Le Conseil fédéral décide, sur le fond, s'il convient d'accorder la protection provisoire et à combien de personnes.

2. L'admission de personnes à protéger ne présuppose pas qu'elles séjourneront durablement en Suisse ; elles retourneront dans leur Etat d'origine ou de provenance dès que la possibilité leur en sera donnée.

3. La procédure est conçue de manière que, contrairement à la solution actuelle de l'admission provisoire de groupes (...), les autorités compétentes en matière d'asile seront dispensées de mener une procédure individuelle, longue et coûteuse. (Message 95.088, Condensé, p. 2).

Le BODS (Mouvement pour une Suisse ouverte, démocratique et solidaire) et la Coordination suisse pour l'asile estiment que cette simplification de la procédure présente aussi un danger : un requérant d'asile appartenant à un groupe au bénéfice d'une protection collective ne pourra pas obtenir l'asile à titre individuel tant que la protection collective n'aura pas été levée. Cet organisme demande donc que les réfugiés de la violence puissent continuer de demander l'asile à titre individuel et que celui-ci leur soit accordé s'ils sont en mesure de prouver avoir fait l'objet de persécutions individuelles. A l'article 66 du projet, le Conseil fédéral prévoit qu'une procédure d'asile ordinaire pourra être entamée « dans des cas exceptionnels ».

Pays sûrs : En 1990, un arrêté fédéral de portée générale a introduit la notion de " pays sûr ", selon laquelle la Suisse n'entre pas en matière sur des demandes d'asile déposées par des ressortissants de pays où le risque de persécutions est inexistant. C'est le Conseil fédéral qui tient la liste de ces pays à jour, ajoutant ou enlevant des Etats selon son appréciation. Cette liste réunit les pays suivants (état en septembre 1996) : Bulgarie, Roumanie, Albanie, Ghana, Sénégal, Gambie, Inde. Pour ce qui est de la Gambie et du Ghana, le Conseil fédéral réexaminera leur statut de pays sûr au printemps 1997. Depuis que cette liste existe, son principe a été repris par quasiment tous les Etats de l'Union européenne.

\section{$\square$ Politique migratoire}

En 1993, le Parlement a chargé le Conseil fédéral de formuler une politique migratoire et d'élaborer les dispositions constitutionnelles dans le domaine, ainsi qu'une loi sur les migrations. Cette politique devrait servir à ralentir encore l'accroissement de la population étrangère et de mieux coordonner les politiques migratoire, de l'asile et des étrangers. 
Afin de disposer d'une base de travail, le Conseil fédéral a commandé un Rapport sur une politique suisse en matière de migrations (qui a été rédigé par Peter Arbenz, ancien directeur de l'ODR). Le rapport a suscité de nombreuses réactions : 72 organismes ont exprimé leur avis lors de la procédure de consultation. Les opinions se recoupent sur quelques points centraux : manque de cohérence entre les divers domaines politiques, insuffisance de la coordination entre les offices fédéraux et consultation insuffisante de l'opinion publique. Mais les positions divergent grandement dans d'autres domaines importants. Ainsi, la Commission fédérale contre le racisme juge que le modèle des trois cercles appliqué dans la politique en matière d'étrangers - et intégré dans la loi sur les étrangers depuis 1995 - constitue une incitation au racisme.

Les idées concernant la procédure à suivre pour formuler une politique migratoire sont parfois diamétralement opposées. C'est dire qu'il ne sera pas facile de réunir un consensus national autour de cette nouvelle politique. Une commission d'experts a d'ores et déjà été chargée de formuler des propositions de cette future politique, qui devraient être présentées au milieu de 1997. L'exigence posée par le Conseil fédéral est la suivante : assurer une meilleure coordination entre les politiques de l'asile, des étrangers, du marché du travail et de développement.

1996 Politique d'asile de la Suisse et questions migratoires, pp. 95-105.

\section{SOURCES}

Message concernant la révision totale de la loi sur l'asile ainsi que la modification de la loi fédérale sur le séjour et l'établissement des étrangers du 4.12.1995 (95.008).

Office fédéral des réfugiés, Statistique en matière d'asile 1995, Liste des pays sûrs, Asylon Aktuelle (éditions de 1995 et de 1996).

DFJP/Office fédéral des réfugiés, Déclaration du conseiller fédéral Arnold Koller à l'occasion de la votation sur l'initiative " contre l'immigration clandestine », 1 décembre 1996.

Muriel Beck Kadima et Jean-Claude Huot, Kirche und Asyl, Legitimer Widerstand im Rechtsstaat ?, Zurich : Institut d'éthique sociale de la FEPS, 1996.

Amnesty International, Amnesty Magazin, Nr. 2, Februar 1996, « Sri Lanka - blinde Rückschaffungspolitik ».

BODS-Rundbreif, Nr. 2, Juni 1996 «Worum es beim neuen Asylgesetz geht », Nr. 3, September 1996 « Abstimmung Asylinitiative ".

Coordination suisse pour l'asile, Asylgesetz : Total überholt, Stellungnahme zur Totalrevision des Asylgesetzes, Argumentenkatalog, Berne.

Parti écologiste suisse, La position des Verts sur la politique de migration, Berne : éd. Parti écologiste suisse, octobre 1996.

Neue Zürcher Zeitung, 15. Mai 1996 « Enge Spielräume in der Migrationspolitik », 11. Juni 1996 « Expertenkommision für Migrationspolitik ». 


\section{FUITES DE CAPITAUX, CORRUPTION, BLANCHIMENT D'ARGENT}

Durant l'automne-hiver 1996-97, la vie politique suisse a été marquée par l'affaire des fonds en déshérence et de l'or nazi, qui a mis la place financière helvétique sur la sellette. Ces événements n'ont pas contribué à améliorer l'image de cette dernière, considérée par certains comme une véritable plaque tournante de l'argent du crime organisé. Pourtant un certain nombre de mesures ont été prises en matière de lutte contre la grande criminalité, au niveau suisse comme au niveau international. En Suisse, le Parlement a accepté la révision sur la loi d'entraide judiciaire internationale, révision trop timide aux yeux de certains magistrats. Le Conseil fédéral a présenté un message sur la lutte contre le blanchiment de l'argent. D'autre part, au niveau international, la Suisse participe aux travaux $d u$ Groupe d'action financière (GAFI) de l'OCDE. Transparency International, organisation non gouvernementale, qui lutte contre la corruption dans les transactions commerciales internationales, a ouvert une section en Suisse.

\section{$\square$ Entraide judiciaire internationale}

Le Conseil fédéral a fixé au 1er février 1997 l'entrée en vigueur de la loi révisée sur l'entraide internationale en matière pénale ${ }^{1}$. Lors de la session d'automne 1996, le Conseil des Etats a accepté de façon définitive la loi révisée sur l'entraide internationale en matière pénale. La principale nouveauté consiste en un unique recours dans le cadre d'une procédure. Seule la décision finale sera attaquable, les recours préventifs n'étant plus possibles. Les juges suisses pourront en outre transmettre spontanément à leurs collègues étrangers des moyens de preuves et des informations à certaines conditions. Cette possibilité d'échapper à la procédure ne s'appliquera toutefois pas au moyen de preuves relevant du secret bancaire. Durant les débats parlementaires, une divergence d'importance est apparue entre les Chambres. Lors de la session de décembre 1995, le Conseil national avait décidé, contre l'avis du Conseil fédéral, d'imposer un délai fixé à neuf mois pour traiter des demandes d'entraide. Le Conseil des Etats n'a pas suivi cette proposition.

Cette révision a été acceptée avec tiédeur par différents milieux. D'une part, les juges d'instruction s'occupant d'entraide se sont dit déçus de la révision adoptée par le Parlement. Dans les cantons les plus concernés (Zurich, Genève et Tessin), dont les banques ont été éclaboussées par des scandales politico-financiers à l'étranger, certains magistrats ont dit n'attendre aucun changement majeur de pratique. Le maintien d'une instance cantonale de recours, dont la décision est susceptible d'être attaquée au Tribunal fédéral demeure, selon eux, le principal obstacle à un traitement rapide des commissions rogatoires envoyées à l'étranger. La Déclaration de Berne ${ }^{2}$, relève les progrès de la révision, notamment en matière de limitation des possibilités de recours à une seule occasion, mais déplore qu'aucune durée de procédure ne soit précisée dans la loi et qu'il n'y ait pas d'élargissement des domaines pour lesquels l'entraide est accordée (évasion fiscale).

1 Loi fédérale sur l'entraide internationale en matière pénale, modification du 4 octobre 1996, in Feuille fédérale, $\mathrm{n}^{\circ} 41$, Volume IV, 15 octobre 1996.

2 La Déclaration de Berne avait rédigé en 1991 un manifeste « Pour une Suisse sans capitaux en fuite », soutenu par plus de $200 \mathrm{ONG}$, qui demandait une limitation de la procédure à six mois, l'élargissement du domaine de compétence pour l'entraide pénale et une procédure d'entraide centralisée au niveau de la Confédération pour les cas de « fuite de capitaux » de chefs d'Etat déchus. 
Le conseiller fédéral Arnold Koller et le ministre français de la justice Jacques Toubon ont signé, fin octobre 1996 à Berne, un accord additionnel à la Convention européenne d'entraide judiciaire $(\mathrm{CEEJ})$ en matière pénale. Le but de cet accord est de simplifier et d'accélérer la transmission des commissions rogatoires entre les deux pays. Ainsi les autorités judiciaires pourront faire leurs demandes d'entraide judiciaire directement au Procureur général auprès de la Cour d'appel compétente ratio loci. Elles n'ont plus besoin de passer par le Ministère français de la Justice. La transmission directe des commissions rogatoires entre juges suisses et français demeure toujours possible dans des cas d'urgence (cf. art. 15, $\S 2, \mathrm{CEEJ})$.

Par ailleurs la Suisse a paraphé, en juillet 1996, un accord d'entraide judiciaire avec le Pérou et un autre avec l'Equateur. Chacun de ces accords prévoit la transmission directe des commissions rogatoires entre les autorités centrales de la Suisse et du pays concerné. Notons que c'est la première fois que ce type d'accord est conclu avec des pays d'Amérique latine.

눝․ 1996 Entraide judiciaire internationale, p. 108.

\section{$\square$ Message sur la lutte contre le blanchissage d'argent}

Le crime organisé est aujourd'hui un des fléaux qui menacent l'économie et la société. Grâce à leurs activités délictueuses (trafic de drogue, commerce des armes, réseaux de prostitution de femmes et d'enfants, etc.), les organisations criminelles disposent de fonds colossaux, qu'elles ne peuvent toutefois pas encore utiliser tant qu'il est possible d'en prouver l'origine. Ce n'est que lorsque cet argent est «blanchi » qu'il peut entrer impunément dans le circuit économique. A partir de ce moment, le pouvoir économique du crime organisé devient exponentiel. L'argent blanchi peut être investi sans éveiller les soupçons et contribue donc à donner à la pègre un vernis de respectabilité. Il y a ainsi un risque réel que des économies entières tombent sous son contrôle.

Depuis plusieurs années, les Chambres et le Conseil fédéral s'efforcent de renforcer la législation pour mieux combattre ces nouvelles formes de criminalité. En moins de dix ans l'arsenal s'est beaucoup étoffé. Le 1er août 1990 sont entrés en vigueur les articles $305^{\text {bis }}$ et $305^{\text {ter }}$ du code pénal (CP) concernant les délits de blanchiment d'argent et de défaut de vigilance en matière d'opérations financières. Cet arsenal pénal a été complété le 1er août 1994. Un second train de mesures de lutte contre le crime organisé a permis la confiscation de toutes les valeurs patrimoniales contrôlées par une organisation criminelle (art. 59, ch. 3 $\mathrm{CP}$ ), a rendu punissable la participation ou le soutien à des organisations criminelles (art. 260 ${ }^{\text {ter }} \mathrm{CP}$ ) et enfin, a introduit dans le code pénal le droit d'annoncer les soupçons (art. $305^{\text {ter }}, 2^{\mathrm{e}}$ al. CP).

Un dernier paquet législatif a été présenté par le Conseil fédéral en juin 1996 afin de compléter les mesures pénales entrées en vigueur en 1990 et 1994 . Dans son message $^{3}$, le Conseil fédéral écrit « la loi sur la lutte contre le blanchissage de l'argent (LBA) impose aux personnes et aux entreprises travaillant dans le secteur financier des obligations de diligence ainsi que des mesures organisationnelles destinées à empêcher le blanchissage. Les intermédiaires financiers sont

${ }^{3}$ Message relatif à la loi fédérale concernant la lutte contre le blanchissage d'argent dans le secteur financier, in Feuille fédérale, $\mathrm{n}^{\circ 37}$, Volume III, 17 septembre 1996. 
en outre tenus d'informer les autorités pénales et de bloquer les valeurs patrimoniales suspectes au premier soupçon fondé de blanchissage. »

Ce projet de loi comble une lacune. Contrairement aux banques, Bourses, négociants de valeurs mobilières, assurances et fonds de placements, les autres intermédiaires financiers n'étaient pas soumis à surveillance fédérale. Le projet de loi étend à l'ensemble des activités non bancaire l'obligation de diligence, qui consiste à vérifier l'identité d'un client, à identifier les ayants droit économiques des valeurs en cause et à garder la trace de leurs transactions. La question de savoir quelles obligations seraient imposées à l'intermédiaire financier qui soupçonne un cas de blanchiment est controversée. De nombreux milieux militaient pour un simple blocage des valeurs suspectes. Le Conseil fédéral a néanmoins opté pour l'obligation d'annoncer les soupçons à un bureau de communication placé au sein de l'Office fédéral de la police, lequel peut saisir les autorités pénales en cas de besoin, et de bloquer immédiatement les valeurs en cause.

Par ailleurs, le Message rappelle que selon des recommandations internationales (Groupe d'action financière sur le blanchiment de capitaux de l'OCDE - GAFI) l'obligation de communication fait partie de la panoplie internationale de lutte contre le blanchiment de l'argent. En mars 1993, la Suisse a été soumise à la vérification de la transposition des recommandations du GAFI. Il avait été alors relevé que la Suisse est bien placée sur le plan international en matière de lutte contre le blanchiment d'argent. Des lacunes avaient été constatées dans le secteur des institutions financières non bancaires. Le rapport du GAFI mentionnait l'avant-projet de la loi sur le blanchiment de l'argent et indiquait clairement que son adoption à bref délai apparaissait comme une nécessité. Cette obligation d'annonce, vigoureusement contestée par les milieux financiers suisses, existe dans l'ensemble des pays d'Europe occidentale et aux Etats-Unis. En novembre 1996, la Commission des affaires juridiques du Conseil national s'est réunie et, à l'unanimité, a voté un texte qui prévoit un durcissement du projet de loi gouvernemental. La Commission propose de punir d'une amende, jusqu'à 200'000 francs, le seul fait de négliger le devoir de communication à l'autorité. Reste à voir si le Parlement suit les propositions de la Commission. Le message du Conseil fédéral est à l'ordre du jour de la session de mars 1997 du Conseil national.

\section{$\square$ Lutte contre la corruption}

\section{Rapport "Contrôle de sécurité et corruption"}

Ces dernières années de nombreux cas de corruption impliquant des hommes politiques et de hauts fonctionnaires ont été dévoilés au public dans des pays industrialisés (Italie, France, Espagne). La Suisse n'a pas été épargnée ; un certain nombre d'affaires, telles celles impliquant Raphael Huber ancien chef des patentes de l'administration zurichoise ou 1'Union suisse du commerce du fromage, ont retenu l'attention des médias et de la justice. Ces différentes affaires démontrent que la corruption n'existe pas seulement dans les pays en développement, mais est un phénomène qui concerne l'ensemble de la société, principalement les domaines de l'administration, de l'économie et de la politique.

En juillet 1995, le conseiller fédéral en charge du département de la Justice, Arnold Koller, a réunit un groupe de travail baptisé « Contrôle de sécurité et corruption ». Dirigé par Urs von Daeniken, les experts - quatre représentants de la Confédération, deux délégués des cantons et un représentant du Parquet zuri- 
chois - ont rédigé un rapport ${ }^{4}$ et des propositions à l'intention du Conseil fédéral, rendu public en novembre 1996. Le Groupe de travail juge « que la situation n'est pas alarmante en Suisse ; au vu des signes négatifs et du contexte économique actuellement défavorable, ainsi que des développements auxquels on assiste à l'étranger, il estime toutefois que des mesures spécifiques sont nécessaires pour lutter efficacement contre la corruption. » De l'avis des experts, les statistiques disponibles - 40 à 50 condamnations pénales par année depuis 1985 - ne sont pas représentatives. Mais des zones d'ombre subsistent et les affaires les plus retentissantes n'ont pas encore été jugées ou sont en procédure d'appel. Les chiffres ne suffisent donc pas à se faire une idée de la situation réelle en Suisse. En conséquence, le Groupe de travail préconise des mesures concrètes de nature répressive et préventive, considérant qu'il est " primordial de combattre simultanément la corruption sur tous les fronts, à savoir, dans l'administration, l'économie et la politique ".

Les recommandations visant à la prévention et à la lutte contre la corruption se résume comme suit :

\ réglementation type de portée générale régissant l'acceptation de dons personnels ;

- établissement d'une liste des fonctions dont les titulaires participent de manière déterminantes aux décisions d'attributions de mandats ou d'achats,

- examen de l'introduction d'un double contrôle (et contrôle interne) dans les domaines de l'administration s'occupant de marché public,

- rotation périodique des responsables,

- création d'une commission permanente interdépartementale, ayant pour mission de suivre et d'analyser les développements de la situation.

Les recommandations en matière de répression comportent notamment des mesures dans les domaines suivants :

๖ pénalisation de la remise de dons à des agents de la fonction publique,

- renforcement du code pénal suisse, la corruption devant être punie plus sévèrement (jusqu'à cinq ans de réclusion) et les délais de prescription prolongés,

- la corruption active de fonctionnaires étrangers devrait être cataloguée dans les actes punissables,

- en coopération avec l'étranger, il y aurait lieu de convenir d'une interdiction de déduire de l'impôt les sommes versées à des fins de corruption ou au titre de pots-de-vin.

Lors de la session d'hiver, le Conseil des Etats a approuvé sans opposition la motion de Kurt Schuele (radical de Schaffhouse) demandant au gouvernement de faire des propositions pour adapter l'arsenal législatif à la lutte contre ce phénomène. En réponse à cette motion Arnold Koller a assuré à la Chambre des cantons que d'ici à la fin 1997, un concept global sur les moyens de renforcer la lutte contre la corruption par le droit pénal serait présenté. Ce concept, qui tiendra compte des recommandations du groupe de travail « Contrôle de sécurité et corruption ", sera alors soumis en consultation.

${ }^{4}$ Schlussbericht des Arbeitsgruppe « Sicherheitsprüfungen und Korruption », Eidgenössischer Justiz- und Polizeidepartement, Oktober 1996. Un résumé du présent rapport est disponible en français et en allemand. 
Déductibilité fiscales des "pots-de-vin »

La pratique fédérale, fondée sur une circulaire de l'administration fédérale des contributions de 1946, selon laquelle les dessous de table versés dans le but d'obtenir des travaux ou des commandes sont déductibles des impôts au titre de frais d'acquisition du revenu, est de plus en plus contestée par certains milieux. Ces derniers considèrent que ce phénomène favorise très concrètement la corruption active, ce d'autant plus qu'il est admis, en ce qui concerne les pots-devin versés à l'étranger, qu'ils sont conformes aux usages commerciaux. Les milieux économiques ne se disent pas opposés à une plus grande morale fiscale, mais ils laissent entendre que ces mesures « touchent aux intérêts vitaux de l'industrie d'exportation en créant un désavantage concurrentiel ». Actuellement, seuls les Etats-Unis punissent expressément la corruption de fonctionnaires étrangers et de plus en plus nombreux sont les pays qui refusent de déduire fiscalement les pots-de-vin. En avril 1996, l'OCDE a encouragé l'ensemble de ses membres a en faire de même.

Une initiative parlementaire de Werner Carobbio, adoptée à une courte majorité au Conseil national en 1995, qui demandait que ces déductions soient abandonnées, a déclenché un processus de réflexion en Suisse. Après un long examen de cette question, la Commission de l'économie et des redevances du Conseil national propose d'interdire la déduction des sommes versées à des fonctionnaires suisses, mais elle compte la maintenir pour les pots-de-vin offerts à des privés ou à des fonctionnaires étrangers.

\section{Autres initiatives de lutte contre la corruption et le crime organisé}

- L'OCDE et la lutte contre la corruption au niveau international

L'OCDE s'est fixé un certain nombre d'objectifs favorisant la lutter contre la corruption au niveau international et plus spécifiquement dans les transactions commerciales. Le principal objectif est de favoriser une bonne gestion des affaires publiques et des entreprises et d'éviter une concurrence déloyale dans les échanges et les investissements internationaux. L'OCDE a entamé ses travaux sur la corruption en 1989 à l'initiative des Etats-Unis. Les Membres ont adopté en 1994 une Recommandation générale qui les enjoint de prendre des mesures concrètes et significatives pour combattre la corruption dans le cadre de transactions commerciales internationales. Une étape supplémentaire a été franchie en avril 1996 avec l'adoption par le Conseil d'une Recommandation sur la déductibilité fiscale des paiements illicites. La Recommandation précise que les Membres qui autorisent la déductibilité devraient réexaminer leur législation en vue d'interdire cette pratique. ${ }^{5}$

1996 Recommandation de l'OCDE pour lutter contre la corruption, p. 110.

- Deux rapports du FMI sur les effets du blanchiment de l'argent

Deux rapports du FMI, publiés en juillet 1996, expliquent que les mouvements de capitaux criminels sont en mesure de déstabiliser les économies des pays dans lesquels ces capitaux sont investis. De plus, ces investissements

5 OCDE, Communiqué de presse du 6 novembre 1996, Déclaration du Secrétaire général de l'OCDE à l'ouverture de la Conférence sur la corruption dans le cadre de transactions commerciales internationales. 
illogiques aux yeux du marché peuvent mettre à néant les politiques économiques menées par les gouvernements. ${ }^{6}$

- «Appel de Genève » signé par sept juges européens

Pour briser les obstacles qui pénalisent leurs actions, sept magistrats européens ${ }^{7}$ ont lancé le 1er octobre 1996 à Genève, un appel dont l'objectif est de proposer l'instauration d'un espace judiciaire européen afin de démanteler les entraves qu'ils rencontrent dans leur lutte contre la corruption. «Pour avoir une chance de lutter contre une criminalité qui profite largement des réglementations en vigueur dans les différents pays européens, il est urgent d'abolir les protectionnismes dépassés en matière policière et judiciaire. Il devient nécessaire d'instaurer un véritable espace judiciaire européen au sein duquel les magistrats pourront, sans entraves autres que celles de l'Etat de droit, rechercher et échanger les informations utiles aux enquêtes en cours » écrivent-ils. Le texte de l'Appel figure à la fin d'un ouvrage ${ }^{8}$ constitué d'entretiens réalisés par le journaliste français Denis Robert avec les sept magistrats, qui expliquent les difficultés rencontrées dans leur travail.

- Transparency International, section suisse

Transparency International, lancée en mai 1993, est une organisation non gouvernementale qui lutte contre la corruption dans les transactions commerciales. Son siège se trouve à Berlin, mais de plus en plus de groupes nationaux se créent. Ainsi Transparency Switzerland ${ }^{9}$, créée en novembre 1995, a pour vocation la prévention de la corruption ainsi que la lutte contre les diverses formes de la corruption et l'explication de ses causes et de ses effets. Elle stimule la réflexion et les débats sur cette question, tout en avançant des propositions de solutions au niveau juridique, politique et économique.

\section{$\square$ Activités du Groupe d'action financière (GAFI)}

La Suisse participe activement aux travaux du Groupe d'action financière sur le blanchiment de capitaux de l'OCDE. Le GAFI a été créé lors du Sommet économique du G-7 qui s'est tenu à Paris en 1989, avec pour mission d'étudier des mesures destinées à combattre le blanchiment de capitaux. En avril 1990, il a publié un rapport de quarante Recommandations dans ce domaine, ces dernières avaient fait l'objet d'une première révision en 1993. Le GAFI regroupe vingt-six Etats $^{10}$ et deux organisations régionales ${ }^{11}$ représentant les principaux centres financiers mondiaux.

Les principales activités de la septième session du GAFI (1995-96) se sont concentrées sur trois axes :

$\checkmark$ La revue des quarante Recommandations de 1990. Plusieurs modifications substantielles ont été convenues par les membres du GAFI. Premièrement, les délits visés en matière de blanchiment ont été étendus au-delà du trafic de stu-

${ }^{6}$ IMF Working Papers von Tanzi, Vito : " Money Laundering and the International System », $\mathrm{n}^{\circ}$ 96/55. Quirk, Peter : " Macroeconomic Implications of Money Laundering ", $n^{\circ}$ 96/66.

7 Bernard Bertossa (Suisse), Edmundo Bruti-Liberati et Gherardo Colombo (Italie), Benoît Dejemeppe (Belgique), Baltazar Garzon Real et Carlos Jimenez Billarero (Espagne), et Renaud Van Ruymbeke (France).

8 Denis Robert, La justice ou le chaos, Paris : Stock, 1996.

9 Transparency Switzerland, Haltbergstrasse 25, 8630 Rüti, tél. 055.241.11.17.

${ }^{10}$ Allemagne, Australie, Autriche, Belgique, Canada, Danemark, Espagne, Etats-Unis, Finlande, France, Grèce, Hong Kong, Irlande, Islande, Italie, Japon, Luxembourg, Norvège, Nouvelle-Zélande, Portugal, Royaume des Pays-Bas, Royaume-Uni, Singapour, Suède, Suisse et Turquie.

${ }^{11}$ Commission européenne et Conseil de coopération du Golfe. 
péfiants. Deuxièmement les déclarations de transactions suspectes sont devenues obligatoires et le champ d'application a été étendu aux entreprises non financières. Parmi les autres modifications figurent les questions des sociétésécrans, l'affinement des obligations en matière d'identification des clients, ainsi que les nouvelles évolutions technologiques, la surveillance des mouvements transfrontaliers d'espèces, la technique de la livraison surveillée et des bureaux de change.

- Suivi de l'application par les pays membres des quarante Recommandations. Une part importante des travaux du GAFI consiste à s'assurer de l'application des quarante Recommandations. Une série de mesures ont été mises au point pour aider les pays membres à y parvenir en les incitant à une autoévaluation. D'autre part les pays sont soumis à une évaluation mutuelle. Chaque année, des pays sont soumis à un examen approfondi; ce fut le tour de la France et de la Suède durant l'exercice 1995-96. Actuellement le GAFI a lancé une deuxième série d'évaluation, la Suisse sera à nouveau analysée en 1997.

- Application des Recommandations pour les pays non membres. En 19951996, le GAFI a adopté une politique d'évaluation de l'application de mesures anti-blanchiment de capitaux par les gouvernements des pays non membre.

1996 Fuites de capitaux, pp. 105-111.

\section{SOURCES}

Loi fédérale sur l'entraide internationale en matière pénale, in Feuille fédérale, n 41, Volume IV, 15 octobre 1996.

Message relatif à la loi fédérale concernant la lutte contre le blanchissage d'argent dans le secteur financier, in Feuille fédérale, $\mathrm{n}^{\circ} 37$, Volume III, 17 septembre 1996.

Schlussbericht der Arbeitsgruppe "Sicherheitsprüfungen und Korruption ", Eidgenössischer Justiz- und Polizeidepartement, Oktober 1996.

Groupe d'action financière sur le blanchiment de capitaux, Rapport annuel 1995-1996, GAFI, 28 juin 1996.

Denis Robert, La justice ou le chaos, Paris : Stock, 1996.

Carla del Ponte, « La Suisse dans la sphère internationale de la criminalité », La vie économique, 2/1996.

Déclaration de Berne, Vers un développement solidaire, $\mathrm{n}^{\circ}$ 137, janvier 1997, Dossier spécial sur la corruption.

Finanzplatz Informationen, 4/1996, "Geldwäscherei - vom Normalfall zur Ausnahme ? ».

Basler Zeitung, 15. April 1996 " Geldwäschereigesetz : Notwendigkeit eines Marschhaltes ».

Le Courrier, 2 octobre 1996 «Genève accueille des juges en colère », 12 décembre 1996 « Le Conseil des Etats active la lutte anticorruption ».

Journal de Genève, 5 octobre 1996 « Lutte contre le crime organisé, la Suisse se muscle, mais...», 29 octobre 1996 « Les juges suisses pourront parler des affaires aux procureurs français », ler novembre 1996 «Taxer les pots-de-vin ? Souhaitable, mais difficilement réalisable ", 12 novembre 1996 " La Suisse veut combattre la corruption».

Neue Zürcher Zeitung, 7. August 1996 « Die Auswirkung der Geldwäscherei, Forderung nach schärferen Massanhmen in zwei IMF-Studien ".

Nouveau Quotidien, 4 octobre 1996 « Les banques suisses commencent à redouter l'argent des dictateurs », ler novembre 1996 «Il faut mettre les pots-de-vin hors la loi », 12 novembre 1996 « Le Conseil fédéral se donnera-t-il les moyens de combattre la corruption », 21 novembre 1996 «Loi anti-blanchissage les politiques balaient les objections des banquiers ».

Tages-Anzeiger, 18. März 1996 « Die Rechtshilfe soll beschleunigt werden», 2. Oktober 1996 «Zaghafte Reform der Rechtshilfe ».

Die Wochenzeitung, 6. September 1996 « Wozdossier - Geldwäscherei ». 


\section{COMMERCE DES ARMES}

Depuis 1987, on constate un recul des dépenses militaires dans le monde. Entre 1990 et 1995, le ratio dépenses militaires/PIB a diminué dans 90 pays et augmenté dans 25 pays. Sur le plan international, 1996 aura été marqué par de nombreuses réunions tendant à limiter ou à interdire l'emploi de certaines armes conventionnelles, notamment les mines antipersonnel.

En Suisse, les Chambres fédérales ont approuvé le 13 décembre 1996 la révision de la loi fédérale sur le matériel de guerre. Cette révision a été marquée par la décision de ne pas inclure les avions Pilatus dans le champ d'application de cette loi, mais d'en soumettre l'exportation aux dispositions de la loi sur le contrôle des biens à usages civil et militaire. La même solution a été adoptée par le Parlement pour les machines et outils exclusivement conçus pour la fabrication ou l'entretien du matériel de guerre. Le Parlement a ainsi réduit le champ d'application de la loi sur le matériel de guerre par rapport au projet présenté par le Conseil fédéral. Un tel assouplissement correspond aux voeux exprimés par les milieux économiques.

\section{$\square$ Exportations suisses d'armes}

Selon le rapport du SIPRI, la vente d'armes conventionnelles dans le monde en 1995 (\$22,8 milliards) ont diminué de 0,2\% par rapport à 1994. Les Etats-Unis, la Russie, l'Allemagne, la Grande-Bretagne et la France sont les cinq principaux exportateurs. La Suisse quant à elle, n'apparaît pas en 1995 dans la liste des 30 principaux exportateurs ; ses exportations d'armes vers tous les pays se sont élevées à 141,17 millions de francs, ce qui représente $0,15 \%$ des exportations totales de la Suisse $(0,23 \%$ en 1994). Selon le Département militaire fédéral, il s'agit du chiffre le plus bas depuis l'entrée en vigueur en 1973 de la loi fédérale sur le matériel de guerre. Entre 1994 et 1995, la valeur des exportations vers les pays en développement a fortement diminué ; il faut toutefois relever que ces chiffres connaissent traditionnellement de fortes fluctuations d'une année à l'autre.

Depuis 1988 la part de la Suisse aux exportations d'armements vers les pays en développement a oscillé entre 0,11 et $0,29 \%$.

\section{$\square$ Révision de la loi fédérale sur le matériel de guerre}

En 1996, les Chambres fédérales ont délibéré sur l'initiative populaire «pour l'interdiction d'exporter du matériel de guerre » ainsi que sur le projet révision de la loi fédérale sur le matériel de guerre que lui avait soumis le Conseil fédéral dans un message du 15 février 1995.

Aussi bien le Conseil national (en mars) que le Conseil des Etats (en octobre) suivirent la proposition du Conseil fédéral de recommander au peuple de rejeter l'initiative soutenue notamment par le parti socialiste et par de nombreuses associations liées au développement et au mouvement pour la paix. Les partisans de l'initiative considèrent qu'une telle interdiction « est une condition de base pour mener une politique de paix cohérente $»^{\prime}$. Les opposants font valoir qu'une telle proposition aurait de graves conséquences économiques, sur le plan de l'emploi.

\footnotetext{
I Arguments et commentaires des initiatives populaires pour le désarmement et pour une interdiction des exportations d'armes, rapport de mai 1991 (voir sources), p.2.
} 
Tableau no 16

Exportations suisses de matériel de guerre 1994/1995 Les 15 principaux pays en développement clients de la Suisse

\begin{tabular}{|c|c|c|c|c|c|}
\hline \multirow[b]{2}{*}{ Pays $^{1}$} & \multicolumn{2}{|c|}{1994} & \multirow[b]{2}{*}{ Pays $^{1}$} & \multicolumn{2}{|c|}{1995} \\
\hline & 1000 fr. & in $\%$ & & 1000 fr. & in \% \\
\hline Arabie Saoudite & $54^{\prime} 232$ & 24.5 & Malaisie & 6'558 & 4.6 \\
\hline Thaïlande & $27 ' 570$ & 12.5 & Inde & 5’318 & 3.8 \\
\hline Inde & $22^{\prime} 969$ & 10.4 & Singapour & 4'345 & 3.1 \\
\hline Singapour & 6'671 & 3.0 & Brésil & 2'129 & 1.5 \\
\hline Malaisie & $5^{\prime} 420$ & 2.5 & Emirats arabes & 2’090 & 1.5 \\
\hline Bahreïn & 3'311 & 1.5 & Argentine & 1'920 & 1.4 \\
\hline Brésil & $1^{\prime} 658$ & 0.8 & Corée du Sud & 1'102 & 0.8 \\
\hline Emirats arabes & 1'637 & 0.7 & Pakistan & 648 & 0.5 \\
\hline Chili & 783 & 0.4 & Maroc & 415 & 0.3 \\
\hline Corée du Sud & 740 & 0.3 & Tunisie & 297 & 0.2 \\
\hline Maroc & 628 & 0.3 & Bulgarie & 190 & 0.1 \\
\hline Egypte & 418 & 0.2 & Egypte & 133 & 0.1 \\
\hline Pérou & 370 & 0.2 & Bahrein & 121 & 0.1 \\
\hline Argentine & 272 & 0.1 & Croatie & 120 & 0.1 \\
\hline Oman & 240 & 0.1 & Oman & 96 & 0.1 \\
\hline $\begin{array}{l}\text { Autres pays en } \\
\text { développement }\end{array}$ & $1 ’ 040$ & 0.5 & $\begin{array}{l}\text { Autres pays en } \\
\text { développement }\end{array}$ & 1'256 & 0.9 \\
\hline $\begin{array}{l}\text { Total des pays en } \\
\text { développement }\end{array}$ & $127 ’ 959$ & 57.9 & $\begin{array}{l}\text { Total des pays en } \\
\text { développement }\end{array}$ & 26739 & 18.9 \\
\hline Pays industrialisés & 93’062 & 42.1 & Pays industrialisés & $114^{\prime} 429$ & 81.1 \\
\hline Exportations totales & $221 ’ 021$ & 100.0 & Exportations totales & $141^{\prime} 169$ & 100 \\
\hline
\end{tabular}

1) Pays de destinations finales.

Source : Département militaire fédéral.

Le débat sur la révision de la loi porta essentiellement sur la définition du matériel entrant dans le champ d'application de la loi. Celle proposée par le Conseil fédéral avait pour conséquence de considérer notamment les avions Pilatus de type PC comme du matériel de guerre ; elle proposait aussi que les machines et les outils exclusivement conçus pour la fabrication ou l'entretien du matériel de guerre devaient aussi entrer dans le champ d'application de la loi.

Les deux Chambres adoptèrent une définition qui place les avions Pilatus hors du champ d'application de la loi. Pour la majorité du Parlement, il s'agit avant tout de ne pas pénaliser une entreprise d'exportation. Il fut décidé de soumettre ces exportations à la nouvelle loi sur le contrôle des biens utilisables à des fins civiles et militaires. A la fin de 1996, un tel embargo concernait une quinzaine de pays. Avec cette modification, les critères définissant les pays pour lesquels les exportations sont soumises à autorisation sont ainsi moins restrictifs que la pratique précédemment adoptée par le Conseil fédéral. 
Sur la question des machines et outils, le Conseil des Etats avait initialement décidé d'inclure ces biens d'équipements dans le champ d'application de la loi. Cela concerne notamment des machines servant à fabriquer des munitions et à forger des tubes de canons. Le Conseil national s'étant prononcé pour leur exclusion du champ d'application de la loi sur le matériel de guerre et leur inclusion dans celui de la loi sur le contrôle des biens, le Conseil des Etats s'est finalement rallié sur ce point au Conseil national. « Les équipements spécifiquement conçus ou modifiés pour un engagement militaire ou pour l'instruction au combat $»^{2}$ ont quant à eux aussi été exclu par le Parlement du champ d'application de la loi sur le matériel de guerre.

La loi fédérale sur le matériel de guerre a pour but de contrôler la fabrication et le transfert de matériel de guerre et de la technologie associée pour respecter les obligations internationales et les principes de la politique étrangère de la Suisse tout en permettant le maintien en Suisse d' une capacité industrielle adaptée aux besoins de sa défense ${ }^{3}$. Les opérations mentionnées ci-dessus doivent donc être soumises à autorisation, et non interdites comme le demande l'initiative populaire.

La loi fédérale sur le contrôle des biens vise quant à elle à contrôler les exportations de matériel à double usage - civil ou militaire - en application de mesures internationales. Elle est destinée à compléter la loi fédérale sur le matériel de guerre. Un article de la loi sur le contrôle des biens autorise le Conseil fédéral à interdire leur exportation vers les pays qui font l'objet d'un embargo international décidé par l'ONU ou l'Union européenne.

Il convient encore de relever que le projet de loi du Conseil fédéral prévoyait (article 21) que, dans ses décisions d'autorisation d'opérations avec l'étranger, le Conseil fédéral devrait prendre en compte notamment la situation des droits de l'homme et les efforts de la Suisse dans le domaine de la coopération au développement dans le pays destinataire. Cette disposition - qui existait, dans la loi en vigueur jusqu'ici - n'a pas été retenue par les Chambres fédérales. La nouvelle loi prévoit par contre - ce qui est nouveau - que le transfert de technologie et le courtage soient - sauf exceptions possibles - soumis à autorisation.

La Communauté de travail des œuvres d'entraide avait pris position en faveur d'une attitude restrictive en matière d'exportation de matériel de guerre, les considérations de politique extérieure devant l'emporter sur les intérêts de l'économie de l'armement.

La Société pour le développement de l'économie suisse (SDES) plaide quant à elle pour une politique qui ne porte pas préjudice à l'économie suisse. Dans le débat parlementaire, un argument souvent avancé pour assouplir les dispositions touchant à l'exportation de matériel de guerre est qu'il fallait cesser de créer des obstacles aux entreprises tournées vers l'exportation. La SDES estimait que le projet du Conseil fédéral remettant « sérieusement en cause la Suisse comme site de production $»$.

2 Article 5 du projet de loi du Conseil fédéral définissant le matériel de guerre.

3 Article premier de la loi fédérale sur le matériel de guerre adoptée par le Parlement le 13 décembre 1996. 


\section{$\square$ Mines antipersonnel}

Conférence d'examen de la Convention des Nations unies sur l'emploi d'armes conventionnelles

En 1995, la Conférence d'examen des Etats parties à la Convention sur les armes classiques qui s'était tenue à Vienne avait abouti à l'adoption d'un protocole sur l'interdiction de l'utilisation d'armes laser aveuglantes. On rappellera que les Etats n'avaient pas pu parvenir à un accord pour renforcer les restrictions internationales actuelles à la production, à la vente, et à l'emploi des mines terrestres et autres armes qui frappent sans discrimination. En 1996, ils ont repris leurs travaux à Genève en deux sessions : du 15 au 19 janvier et du 22 avril au 3 mai.

1996 Commerce des armes et développement, pp. 112-119.

Au terme de ces nouvelles délibérations, quelques petites améliorations ont été apportées au protocole II de la Convention sur les mines terrestres notamment par l'extension de son applicabilité à la fois aux conflits armés internationaux et non internationaux. Cependant, les nouvelles règles limitant l'utilisation des mines (par exemple en exigeant qu'elles soient munies d'un dispositif d'autodestruction), sont faibles et complexes.

Le Comité international de la Croix-rouge (CICR) et les nombreuses organisations non gouvernementales qui avaient appelé à une interdiction totale des mines antipersonnel se sont déclarés déçus des résultats de cette conférence. Elles ont réaffirmé que, dans la pratique, il est impossible de réglementer l'utilisation de ces mines et que l'interdiction totale est la seule solution. Au moment de la dernière session de la Conférence de révision, près de 30 pays s'étaient prononcés en faveur d'une telle interdiction.

Dans le contexte du débat international sur les mines antipersonnel, le CICR avait fait réaliser un sondage d'opinion dans 21 pays. Le pourcentage des personnes interrogées (500 à 1000 par pays) favorable à une interdiction totale des mines antipersonnel s'échelonne de $92 \%$ au Danemark à 58\% au Japon ; pour la Suisse, ce taux est de $88 \%$. Une étude menée également à la demande du CICR par des militaires de haut rang montre :

๖ qu'il est difficile, sinon impossible même pour des armées modernes professionnelles d'utiliser les mines antipersonnel en respectant les règles du droit international ;

a que les coûts humains et sociaux qu'elles occasionnent sont sans commune mesure avec leur efficacité militaire faible et souvent négligeable.

\section{La Conférence d'Ottawa pour l'interdiction totale des mines antipersonnel}

Les résultats limités de la Conférence d'examen (voir ci-dessus) incitèrent 50 Etats ainsi que des organisations internationales et des ONG à se réunir à Ottawa en octobre 1996 à l'invitation du Canada pour mettre en marche un processus devant conduire à l'interdiction totale des mines antipersonnel. Cette conférence aura servi à renforcer la volonté politique des pays participants de faire progresser rapidement ce dossier. La Conférence a abouti à trois résultats : i) la création d'un " groupe d'Ottawa » réunissant 50 Etats qui s'engagent à collaborer à l'interdiction et l'élimination des mines antipersonnel; ii) l'adoption d'un programme d'action à l'échelle globale et régionale et iii) l'invitation par le Gouvernement canadien aux ministres des Affaires étrangères à signer un traité d'in- 
terdiction totale en décembre 1997. La Déclaration d'Ottawa contient aussi un engagement à accroître les ressources pour les opérations de déminage, l'assistance aux victimes ainsi que pour les activités de prévention. La prochaine réunion aura lieu en Belgique en juin 1997.

La Suisse a participé activement à cette conférence et a proposé d'accueillir une prochaine réunion du " groupe d'Ottawa ».

\section{Position de la Suisse sur les mines antipersonnel}

En septembre 1995, la campagne nationale contre les mines antipersonnel déposait une pétition munie de 150'000 signatures. En octobre 1995, le Département militaire fédéral annonçait que l'armée suisse renonçait à l'utilisation de mines antipersonnel autres que celles actionnées par un observateur. En janvier 1996 à Genève, la Suisse se prononçait pour une interdiction totale.

En décembre 1996, le Parlement acceptait d'inclure les mines antipersonnel dans les articles de la loi qui interdisent les armes nucléaires, biologiques et chimiques. La définition des mines antipersonnel finalement retenue est plus restrictive que celle qu'avait initialement proposé le conseiller national John Dupraz de Genève ; les mines servant à la défense anti-char mais pouvant aussi être déclenchée par des personnes sont ainsi exclues du champ de la loi.

\section{$\square$ Cohérence des politiques à l'égard des pays en développement et commerce des armes}

Dans le contexte du débat parlementaire sur l'initiative populaire pour l'interdiction d'exporter du matériel de guerre ainsi que sur la révision de la loi fédérale sur le matériel de guerre, des œuvres d'entraide ont mis en relation les exportations suisses de matériel militaire et la politique extérieure de la Suisse. Dans un dossier intitulé « Le développement a besoin de la paix », Pain pour le prochain a en particulier signalé des « incohérences » avec des principes de la politique étrangère telles que «1'exportation d'armes vers des pays qui violent les droits de l'homme et où règne un climat de guerre civile » (Birmanie, Turquie, Nigéria). Sont aussi signalées les exportations d'armes de la Suisse vers l'Inde et le Pakistan, pays entre lesquels règne une tension et qui sont tous deux pays de concentration de la coopération suisse au développement.

Ce sont les avions Pilatus utilisés par l'armée birmane dans des opérations militaires qui ont particulièrement retenu l'attention des œuvres d'entraide suisses, en particulier de Swissaid et de Caritas qui soutiennent des programmes d'aide à des personnes déplacées dans le contexte de ce conflit. La solution adoptée par les Chambres fédérales permettra désormais d'interdire de telles exportations dans la mesure où la Birmanie demeure soumise à un embargo international.

Sur le même thème, le Conseil national a, sur proposition du Conseil fédéral, rejeté en mars 1996 une motion Ziegler (soc. GE) demandant d'interrompre la livraison de pièces de rechange pour des PC 7 au Mexique en raison de l'engagement de ces avions dans la province du Chiapas.

Par ailleurs, le Tribunal fédéral a considéré que trois dirigeants de 1'entreprise Von Roll s'étaient rendus coupables de violation de la loi sur l'exportation de matériel de guerre dans l'affaire de la livraison à l'Irak de matériel devant servir à la construction d'un «super-canon ». Ils ont ainsi été condamnés à une peine d'un mois de prison avec sursis. 
Message concernant l' initiative populaire "pour l'interdiction d'exporter du matériel de guerre " et la révision de la loi fédérale sur le matériel de guerre du 15 février 1995, in Feuille fédérale, vol. I, 1995.

Message concernant la loi fédérale sur le çontrôle des biens utilisables à des fins civiles et militaires du 22 février 1995, in Feuille fédérale, vol. I, 1995.

Bulletin officiel de l'Assemblée fédérale, Conseil national et Conseil des Etats, Berne, sessions de 1996.

Comité romand des initiatives pour la paix et le désarmement, Arguments pour ou contre l'interdiction d'exporter du matériel de guerre, février 1996.

Pain pour le prochain, Repères 2/96, «Le développement a besoin de paix », Berne et Lausanne : PPP, 1996.

Parti socialiste suisse et Comité pour le désarmement et pour l'arrêt des exportations d'armes, Arguments et commentaires des initiatives populaires pour le désarmement et pour une interdiction des exportations d'armes, Berne, mai 1991, 48 p. Société pour le développement de l'économie suisse (SDES), Opinion, n 38, 17 septembre 1996.

Société pour le développement de l'économie suisse (SDES), Documentation, n 41, 8 octobre 1996.

Fonds monétaire international, Bulletin du FMI, 10 juin 1996.

Journal de Genève, 29 novembre 1996.

Neue Zürcher Zeitung, 2. und 16. Februar 1996, 6. und 7. März, 3. und 7. Oktober 1996, 26. November 1996, 5. Dezember 1996.

Tages Anzeiger, 31. Mai 1996.

1996 Commerce des armes et développement, pp. 112-119.

\section{RATIFICATION DE LA CONVENTION DES NATIONS UNIES RELATIVE AUX DROITS DE L'ENFANT}

En décembre 1996, au terme d'un triple va-et-vient entre les deux Chambres, la Suisse était enfin prête à ratifier la Convention des Nations Unies relative aux droits de l'enfant.

Le Message du Conseil fédéral de juin 1994 recommandait la ratification de la Convention par la Suisse. La question a finalement été débattue au Parlement pendant les sessions parlementaires de juin (Conseil des Etats), d'octobre (Conseil national) et de décembre 1996 (navette entre les Chambres). Lors de la session d'été, au mois de juin, le Conseil des Etats avait accepté l'idée d'une ratification avec cinq réserves (35 voix contre 4), malgré l'opposition d'une minorité de députés de la droite. Dans son message, le Conseil fédéral en avait proposé quatre : le droit au regroupement familial, qui n'est pas prévu pour les saisonniers, les étudiants et les requérants d'asile; le droit de l'enfant à acquérir la nationalité ; la séparation des jeunes et des adultes dans les établissements pénitenciers (les prisons ne sont pas différenciées dans certains cantons); et les garanties en matière de procédure pénale (la gratuité de l'assistance ne libère pas définitivement le bénéficiaire des frais qui en résultent ; la séparation entre l'autorité d'instruction et celle du jugement n'est pas garantie dans tous les cantons). Le Conseil des Etats avait ajouté une cinquième réserve proposée par le démocrate-chrétien Hans Danieth concernant l'autorité parentale (art. 5 de la Convention). Les milieux conservateurs et nationalistes soutenaient cette réserve, craignant « la mainmise des Nations Unies sur des questions qui concernent prioritairement les relations à l'intérieur de la cellule familiale. »

Le $1^{\text {er }}$ octobre, le Conseil national acceptait la ratification (116 voix contre 46) avec les quatre réserves du Conseil fédéral, mais en refusant celle proposée par le Conseil des Etats. Lors de la session d'hiver, après plusieurs navettes entre les deux Chambres, le Conseil national a admis, de guerre lasse, le 12 décembre 
1996, la cinquième réserve du Conseil des Etats. Au nom de la Commission des affaires juridiques du Conseil national, Francine Jeanpêtre a souligné que se ralliement intervenait dans le seul but de ne plus retarder la ratification de la Convention.

En mars 1996, 86 ONG suisses de défense des droits de l'enfant et de développement avaient demandé (une fois de plus) « une ratification le plus rapidement possible ». Après la décision du Parlement, elles ont salué la ratification de la Convention, tout en regrettant vivement ces réserves. Le dépôt des instruments de ratification a eu lieu au printemps 1997, auprès du Secrétaire général de l'ONU. La Suisse devient officiellement le $188^{e}$ pays à ratifier la Convention et comble un retard puisqu'elle faisait partie des cinq derniers Etats (avec les EtatsUnis, les Emirats arabes unis, Oman, les Iles Cook et la Somalie) à ne pas l'avoir ratifiée. En Suisse, la Convention devrait permettre des améliorations dans divers domaines : droit d'être entendu de l'enfant dans des procédures le concernant, comme le divorce ; prises en considération de l'intérêt juridique prépondérant de l'enfant dans les actions des pouvoirs publics ; meilleure intégration des enfants handicapés, adoptés ou réfugiés. La ratification de la Convention permettra également à la Suisse de disposer d'un instrument juridique universellement accepté à l'appui de ses interventions en faveur du respect des droits de l'enfants dans le monde.

1996 Convention relative aux droits de l'enfant, pp. 83-87.

Journal de Genève, 6 juin 1996 "Malgré une guérilla juridique, les Etats acceptent la Convention sur les droits de l'enfant », 2 octobre 1996 « La Suisse pourra ratifier la Convention sur les droits de l'enfant ».

La Liberté, 10 septembre 1996 « Les enfants du monde. attendent que la Suisse ratifie leur convention », 14 décembre 1996 « La Suisse enfin prête à ratifier la Convention des droits de l'enfant ».

Neue Zürcher Zeitung, 6. Juni 1996 « Das Uno-Kinderrechtsabkommen », 7. Juni 1996 " Der Ständerat ratifiziert Kinderrechtskonvention », 10. September 1996 « Die Schweiz und die Uno-Konvention », 2. Oktober 1996 « Ja zur Kinderrechtskonvention der UNO ».

Nouveau Quotidien, 10 septembre 1996 « Droit de l'enfant : la Suisse se hâte lentement ».

Tages Anzeiger, 5. Juin 1996 « Kinder sind die besten Experten». 